\title{
Economic Integration with Endogenous Technological Change in Knowledge-based Services: A New Economy
}

\author{
Sang-Chul Yoon \\ Sogang University
}

\begin{abstract}
This paper introduces a more sophisticated two-sector model of endogenous long-run growth without scale effects. The world economy consists of two similarly developed countries both of which are capable of producing manufacturings and services. The knowledge-based services as intermediate inputs are used most intensively in the service sector and in the 'service' functions of manufacturing sector. In order to highlight the implications of economic integration for long-run performance in the presence of digital markets, we conduct a sequence of comparisons of a country's equilibrium growth path under the alternative scenarios of economic isolation and international integration. In this paper, we seek to reformulate the idea of Grossman and Helpman model to provide in further detail the nexus of increased productivity in the advanced economies through the digital marketplaces in the 1990s.
\end{abstract}

•JEL Classfications : F12, F15, O33

-Key words : Economic integration, Growth without scale effects, Intraindustry trade, Knowledge-based services, New economy

\section{Introduction}

In the recent years the production of goods and services is becoming more and more knowledge-intensive -- more science-intensive via the better use of existing

\footnotetext{
*Corresponding address: Professor Sang-Chul Yoon, Department of Economics, Sogang University, 1, Shinsu-Dong, Mapo-Gu, Seoul 121-742, Korea. Tel: +82-2-705-8503, Fax: +82-2-705-8497, E-mail: syoon@ sogang.ac.kr

(C2004-Center for International Economics, Sejong Institution, All Rights Reserved.
} 
stocks of scientific knowledge (OECD, 1999). In particular, the new kinds of knowledge-based services are central to the current wave of technical change. ${ }^{1}$ Knowledge-based services whose primary input is human creativity include a wide range of services such as softwares, databases, literary works, programs, graphics, designs, images and art works, music and other services which are created, collected, processed and enhanced by human knowledge. With the advent of digital marketplaces such as the Internet, the World Wide Web, the browser and electronic commerce, the more fluid flows of knowledge within and across national borders, the more the knowledge stock is enhanced and the more productivity-enhancing new knowledge-based services each economy can develop. These knowledge-based services are often produced with dramatic and historically unprecedented increasing returns to scale. That is, the knowledgebased services have cost structures characterized by very low variable costs relative to fixed costs, thus average costs decline as production increases (Wolfson, 1999).

All sectors of the economy are experiencing significant changes in the way manufacturings and services are produced and delivered as a result of the increased diffusion and use of knowledge-based services. ${ }^{2}$ The knowledge-based services as intermediate inputs have become an important field of corporate investments in all sectors. In the service sector, investments in knowledge-based services are especially important. In most of the dynamic service industries, investments in knowledge-based services are larger than in the manufacturing sector. That is, services play a leading role in the adoption of information technology. In manufacturings that are adopting information technology and restructuring to exploit the new technology, it is generating new efficiencies by reducing procurement costs and improving supply chain managements. However, the Internet and E-commerce appear to be able to make a substantial contribution to economic growth, particularly in service industries (Ark, Monnikhof and Mulder, 1999; Licht and Moch, 1999; Lehr and Lichtenberg, 1999; OECD, 2000).

We introduce a simple dynamic two-sector general equilibrium model of economic integration with endogenous technological change in knowledge-based

\footnotetext{
${ }^{1}$ As in Choi and Whinston (2000), knowledge-based services are defined as services that can be produced, transferred, or exchanged in digital form. Using technologies, knowledge-based services can be assembled, revised and packaged in almost an infinite number of ways and delivered instantly via the network when and how they are needed.
}

${ }^{2}$ In this analysis, knowledge-based services and intermediate services will be used interchangeably. 
services. In the specific model, we examine a general functional relationship of the knowledge production function and confirm that the process of endogenous innovation can be self-sustaining with only linear accumulation of knowledge capital. Based upon this confirmation, we conduct more sophisticated models of balanced endogenous growth without scale effects with a parameterized version of skilled labor in the knowledge production function. In this analysis the innovating firm incurs resource costs to introduce new intermediate services. Forwardlooking potential innovators conduct R\&D and enter the product market whenever profit opportunities exist. The world economy consists of two similarly developed countries both of which are capable of producing manufacturing goods and services. The knowledge-based services as intermediate inputs are assumed to be produced under conditions of increasing returns to scale with inherent endogenous technological change property. The intermediate services are used most intensively in the service sector and in the 'service' functions of manufacturing sector. Attention has focused on a sequence of comparisons of a country's equilibrium growth path under the alternative scenarios of economic isolation and international integration which can be achieved by increasing trade in knowledgebased services or by increasing flows of knowledge. In the new economy, the digital marketplaces such as Internet lead to a new intra-industry trade in knowledge-based services and the fluid flow of knowledge that have played very important role in facilitating further endogenous growth in the 1990s.

The literature on endogenous technological change is initiated by Romer (1990) and extended by Grossman and Helpman (1991), Aghion and Howitt (1992), and others. These literature imply that growth rates should be monotonically increasing in the level of resources devoted to $R \& D$. However, such a prediction receives little support empirically. Therefore, subsequent endogenous growth models have attempted to eliminate the growth effect of scale. Jones (1995), Segerstrom (1998), and others follow a strategy that leads to a model in which long-run per capita growth is proportional to the rate of population growth. Young (1998), Howitt (1999), and others suggest that an increase in scale increases the number of products available in direct proportion, leaving the amount of research effort per sector unchanged, and therefore growth unchanged. This paper examines a more sophisticated model of endogenous long-run growth without scale effects with a parameterized version of skilled labor in the knowledge production function. The model is newly approached to eliminate scale effects in earlier R\&D-driven endogenous growth model. Important contributions to 
economic integration and endogenous growth include Rivera-Batiz and Romer (1991) and Grossman and Helpman (1991, ch.9). In this analysis, we seek to reformulate the idea of Grossman and Helpman model into a dynamic two-sector general equilibrium model without scale effects to highlight the implication of newly evolving economic integration for long-run performance in the presence of digital marketplaces in the 1990s.

The paper proceeds as follows: Section 2 presents the basic model and specifies its underlying assumptions. Specially, a general functional relationship of knowledge production function is examined. Section 3 discusses a balanced endogenous growth without scale effect. In particular, by allowing for sustained growth, a parameterized version of skilled labor in the knowledge production function is examined. Section 4 conducts a sequence of comparisons of a country's equilibrium growth path under the alternative scenarios of economic isolation and integration. Section 5 concludes the paper.

\section{Basic Model}

In this section we introduce three sectors into the model: two final sectors (a manufacturing sector and a service sector) under competitive conditions and an intermediate service sector under increasing returns to scale. Monopolistic competition prevails in the intermediate service market. The number of intermediate services can be augmented as a result of $R \& D$. The productivity of skilled labor in $R \& D$ depends on the cumulative knowledge in the $R \& D$ technology. The economy is endowed with fixed stocks of two primary factors, unskilled labor, $L$, and skilled labor, $S$. Both are assumed to be physically immobile internationally. The source of economic growth in this model is the introduction of new intermediate services as a result of $R \& D$, which in turn generates dynamic increasing returns to scale in both the production of two final sectors and R\&D.

\section{A. Consumers}

We consider the economy in which the representative consumer has a particular time-separable intertemporal utility function of the form

$$
U=\int_{0}^{\infty} e^{-\rho t}\left[\mu \log c_{x}+(1-\mu) \log c_{y}\right] d t, \text { for } \in(0,1)
$$

where $\rho$ is the constant subjective discount rate, $c_{x}$ is consumption of the final 
services and $c_{y}$ consumption of the manufacturing goods. This form implies constant expenditure shares $\mu$ and $1-\mu$ on service $X$ and good $Y$. Thus, given total expenditure at time $t, E(t)$ and prices, the instantaneous demand functions are $c_{x}=\mu E / p_{x}$ and $c_{y}=(1-\mu) E / p_{y}$, where $p_{x}$ is the price of the service and $p_{y}$ the price of the manufacturing good.

In order to solve for the time pattern of expenditures that maximizes $U$, the consumer must satisfy an intertemporal budget constraint as follows

$$
\int_{t}^{\infty} e^{-[R(\tau)-R(t)]} E(\tau) d \tau=\int_{t}^{\infty} e^{-[R(\tau)-R(t)]} I(\tau) d \tau+B(t)
$$

where $I(\tau)$ is the consumer's factor income in period $\tau, B(t)$ is the value of his accumulated assets at time $\mathrm{t}$, with $B(0)=0$, and $R(\tau)$ is the cumulative interest factor through time $\tau$. We assume that the consumer can borrow or lend freely on a capital market with instantaneous rate of interest $\dot{R}(\tau){ }^{3}$

From the first-order condition for maximizing $U$ subject to (2) at $t=0$, we get that the optimal path for expenditure follows

$$
\frac{\dot{E}}{E}=\dot{R}-\rho
$$

This condition holds for every consumer and also for aggregate spending.

\section{B. Producers}

\section{Functional Forms in the Manufacturing Sector}

The manufacturing good is assumed to be produced subject to constant return to scale using unskilled and skilled labor, and a continuum of intermediate services. The intermediate services are assumed to be symmetric but imperfect substitutes in the production of the services and manufacturings. The production function of $Y$ is assumed to take a sort of modified Cobb-Douglas appearance as follows.

$$
Y=G^{y}\left(L_{y}, S_{y}, \Gamma_{y}\right)=L_{y}^{1-\beta-\gamma} S_{y}^{\beta} \Gamma_{y}^{\gamma}
$$

where $\Gamma_{y}$ is an index of aggregate intermediate services used in sector $Y, S_{y}$ is employment of skilled labor, and $L_{y}$ is employment of unskilled labor in the production of $Y$. Let $\phi_{y}(w, v, q(w, v))$ be the unit cost in production $Y$. Its price, which equals marginal cost, satisfies

\footnotetext{
${ }^{3}$ In this analysis, an overdot represents a time derivative.
} 


$$
P_{y} \equiv n^{\sigma} p_{y}=\phi_{y}(w, v, q(w, v))
$$

where $w$ is the reward to unskilled labor, $v$ the reward to skilled labor, and $q$ the price of any intermediate service. ${ }^{4}$

\section{Functional Form in the Service Sector}

The final service is produced by a continuum of intermediate services subject to constant returns to scale:

$$
X=G^{x}\left(\Gamma_{x}\right)=\Gamma_{x}
$$

where $\Gamma_{x}$ is an index of aggregate intermediate services used in sector $X$. Let $\phi_{x}(q(w, v))$ be the unit cost in production $X$. Its price, which equals marginal cost, satisfies $^{5}$

$$
P_{x} \equiv n^{\sigma} p_{x}=\phi_{x}(q(w, v))
$$

\section{Functional Form in the Intermediate Service Sector}

The intermediate services form a continuum of horizontally differentiated products. We assume that for a given aggregate quantity of intermediate services used in final production, output is higher, the greater is the diversity in the set of input used. This specification captures the productivity gains from increasing degrees of specialization in the production of final goods and services. In particular, we assume that this index of aggregate intermediate services use for sector $j, j=x, y$ is given by

${ }^{4}$ Since all intermediates are priced similarly, producers of manufacturing goods will use equal quantities of each one. If $Z_{y}=n z_{y}$ is the aggregate quantity of intermediates employed in the production of manufacturing goods, then by (8), $\Gamma_{y}=n^{(1-\alpha) / \alpha} Z_{y}$. Substituting (8) into (4), we have

$$
Y=n^{\sigma} \bar{Y}
$$

where $\bar{Y}=L_{y}^{1-\beta-\gamma} S_{y}^{\beta} \Gamma_{y}^{\gamma}$ and $\sigma=\gamma(1-\alpha) / \alpha$. Producers of manufacturing goods maximize profits by setting its price equal to marginal cost. This implies

$$
p_{y}=n^{\sigma} \phi_{y}(w, v, q(w, v)) .
$$

${ }^{5}$ Since all intermediates are priced similarly, producers of services will use equal quantities of each one. If $Z_{x}=n z_{x}$ is the aggregate quantity of intermediates employed in the production of services, then by (8), $\Gamma_{x}=n^{(1-\alpha) / \alpha} Z_{x}$. Now substituting (8) into (6), we have

$$
X=n^{\sigma / \gamma} \bar{X}
$$

where $X=Z_{X}$ and $\sigma=\gamma(1-\alpha) / \alpha$. Producers of services maximize profits by setting its price equal to marginal cost, which yields

$$
p_{y}=n^{-\sigma / \gamma} \phi_{x}(q(w, v))
$$




$$
\Gamma_{j}=\left[\int_{0}^{n} z_{j}(\xi)^{\alpha}\right]^{\frac{1}{\alpha}}
$$

where $0<\alpha<1$ is a positive monotone transformation of the elasticity of substitution, and $z_{j}(\xi)$ is the amount of intermediate service $\xi$ used in the production of service $X$ and manufacturing good $Y$, and $n(t)$ is the measure of the number of varieties available at time $t$.

The intermediate service is assumed to be produced under zero profit in a present value sense, and the input requirements for $R \& D$ do not vary with the number of innovating firms. We shall assume that costs of producing intermediate services comprise two parts, fixed development costs and variable production costs. Let $\phi_{z}(w, v)$ be the unit cost in production and $\phi_{n}(v)$ the cost of developing a blueprint. These costs are the same for all intermediate services, regardless of whether or not the variety has previously been introduced by another innovating firm. Then $\phi_{n}(\cdot)$ is the fixed cost and $\phi_{z}(\cdot)$ is the average and marginal variable cost for all firms in this intermediate service sector. There exists an unbounded set of potential varieties of these intermediate services. Hence, it will never be rational for a producer to develop an already existing intermediate services, and each innovator enjoy monopoly power in the production of his particular variety for the indefinite future. A producer of an existing blueprint faces at time $t$ a measure $n(t)$ of competitors who have developed blueprint in the past.

Our analysis employs a general functional relationship of the knowledge production function by parameterizing our R\&D technology. Specially, a flow of new knowledge is $\dot{n}$ formed by

$$
\dot{n}=\frac{1}{a_{s_{n}}} n^{\theta} S_{n}^{\eta} \text { with } \theta>0, \eta>0
$$

where $a_{s_{n}}$ is inverse productivity parameter, $n$ is the current stock of knowledge capital, $S_{n}$ is aggregate employment of skilled labor in the R\&D sector. By rewriting equation (9), we get the growth rate of knowledge as follows

$$
\frac{\dot{n}}{n}=\frac{1}{a_{s_{n}}} n^{\theta-1} S_{n}^{\eta}
$$

In this analysis, it is assumed that monopolistic competition prevails in the intermediate service market. In the monopolistic competition equilibrium, output per variety $z_{j}(\xi)=z_{j}$ and prices $q(\xi)=q$ for all $\xi \in[0, n(t)]$ satisfy 


$$
\alpha q=\phi_{z}(w, v) \text { with } \alpha=\left(1-\frac{1}{\varepsilon}\right)
$$

where $\varepsilon$ is the price elasticity of demand for intermediate services $z$. The resulting operating profits per variety are

$$
\pi=(1-\alpha) q\left(Z_{x}+Z_{y}\right) / n
$$

where $Z_{j}=n z_{j}$ is the aggregate quantity of intermediate services employed in the production of the two final sector $X$ and $Y$.

With free entry, the present value of future operating profits must equal the current cost of R\&D. Hence, each new variety breaks even, that is,

$$
\int_{t}^{\infty} e^{-[R(\tau)-R(t)]} \pi(\tau) d \tau=\phi_{n}(v(t))
$$

Differentiating this zero-profit condition with respect to $t$, we find

$$
\pi+\dot{\phi}_{n}=\phi_{n} \dot{R}
$$

This implies equilibrium in the capital market. Since knowledge is a free input to each innovating firm, the cost of knowledge-based service development can be obtained by the equation $\Pi \equiv \dot{n} p_{n}-v S_{n}=0$ and equation (9) as follows

$$
p_{n} \equiv \phi_{n}(v)=\frac{a_{S_{n}} v}{\eta n^{\theta} S_{n}^{\eta-1}}
$$

where $p_{n}$ is the price of any blueprints.

Equations (5), (7), (11), and (15) describe the equilibrium relationships between product and factor prices.

\section{Balanced Endogenous Growth without Scale Effect}

There are only two primary factors - unskilled and skilled labor- and there are fixed input-output coefficients. As in Chang (1973), let $a_{i j}$ be the input-output coefficient denoting the units of the $i$ th factor required in producing one unit of the $j$ th good, $i=S, L$, and $j=x, y, z, n$, and define the total input-output coefficients $b_{i x} \equiv a_{i x}+a_{i z} a_{Z_{x}}$.

In Appendix we first examine some of the equilibrium conditions under a general functional relationship of knowledge production function as specified above. As a consequence of the Appendix, we have the following proposition.

Proposition 1 In a general functional relationship of the knowledge production 
function formed by parameterizing such a $\dot{n}=\left(1 / a_{S_{n}}\right) n^{\theta} S_{n}^{\eta}$, with $\theta>0, \eta>0$, the process of endogenous innovation can be self-sustaining with only linear accumulation of knowledge capital of $\theta=1$.

First of all, we examine the case of a nonlinear relationship between cumulative research and the stock of knowledge capital in Appendix. If $\theta<1$, then the contribution of research to general knowledge capital eventually becomes too small to preserve the private incentive for service development. If $\theta>1$, then growth explodes at an ever increasing rate. With only the specialization of $\theta=1$, we find that the process of endogenous innovation can be self-sustaining (see Jones 1999).

In this section we examine a balanced endogenous growth $(\theta=1)$ without scale effect $(0<\eta<1)$. Specially, we parameterize the skilled labor devoted to R\&D in the knowledge production function, and thus allow for concavity in knowledge production. Then the knowledge creation function, which takes the form

$$
\dot{n}=\frac{n}{a_{S_{n}}} S_{n}^{\eta}
$$

where we take the stock of knowledge to be proportional to cumulative experience in $R \& D$.

Since knowledge is a free input to each innovating firm, the cost of a knowledge-based service development can be written by using equation $\Pi \equiv \dot{n} p_{n}-v S_{n}=0$ and equation (16), as follows

$$
p_{n}=a_{S_{n}} v / \eta n S_{n}^{\eta-1}=\phi_{n}
$$

Equations (5), (7), (11) and (17) describe the equilibrium relationships between product and factor prices.

\section{A. Integrated Equilibrium}

The unskilled labor market clearing condition is

$$
b_{L_{x}} \mu \frac{E}{P_{x}}+b_{L_{y}} \frac{(1-\mu) E}{P_{y}}=L
$$

and the skilled labor market clearing condition is

$$
\left(a_{S_{n}} \frac{\dot{n}}{n}\right)^{\frac{1}{n}}+b_{S_{x}} \mu \frac{E}{P_{x}}+b_{S_{y}} \frac{(1-\mu) E}{P_{y}}=S
$$

with $b_{L_{x}}=a_{L_{z}} a_{Z_{x}}, b_{S_{x}}=a_{S_{z}} a_{Z_{x}}, b_{L_{y}}=a_{L_{y}}+a_{L_{z}} a_{Z_{y}}$ and $b_{S_{y}}=a_{S_{y}}+a_{S_{z}} a_{Z_{y}}$. Since 
the cost of a blueprint is $a_{S_{n}} v / \eta n S_{n}^{\eta-1}$, the free entry condition implies

$$
a_{S_{n}} v / \eta n S_{n}^{\eta-1} \geq \phi_{n}
$$

with equality whenever $\dot{n}>0$.

Using the pricing equations (5) and (7), the skilled labor market clearing condition (19), and the free entry condition (20), we can prescribe the evolution of the number of varieties with a normalization of prices that $E(t)=1$ for all $t$ as follows

$$
\frac{\dot{n}}{n}=\frac{1}{a_{S_{n}}}\left[S-\Lambda \eta^{-1}(h S)^{1-\eta} / n \phi_{n}\right]^{\eta} \text { if } \phi_{n}>\frac{\Lambda}{n S \eta(h S)^{\eta-1}}
$$

From these new variables, $\Phi \equiv 1 / n \phi_{n}$ and $\dot{g} \equiv \dot{n} / n$, we can rewrite (21) as

$$
g=\frac{1}{a_{S_{n}}}\left[S-\Lambda \eta^{-1}(h S)^{1-\eta} \Phi\right]^{\eta} \text { if } \Phi<\frac{S \eta(h S)^{\eta-1}}{\Lambda}
$$

Combining (A10) with (A11), we have

$$
\frac{\dot{\Phi}}{\Phi}=(1-\alpha)[\mu+\gamma(1-\mu)] \Phi-g-\rho
$$

We have thus reduced the system of equilibrium relationships to a single differential equation, (23) plus one side condition, (22). We proceed now with a diagrammatic analysis of the equilibrium dynamics.

In Figure 1, the curve $S S$ depicts (22). ${ }^{6}$ This equation, which must be satisfied at every moment in time, is a constraint on resource use. The higher is the rate of innovation, the greater is employment in R\&D. Therefore, a smaller amount of skilled labor remains for two final sectors, and so the supply of two final sectors

${ }^{6}$ Here

$$
\frac{d \Phi}{d g}=-\frac{a_{S_{n}}\left(S-\Lambda \eta^{-1}(h S)^{1-\eta} \Phi\right)^{1-\eta}}{\Lambda(h S)^{1-\eta}}<0
$$

and

$$
\begin{aligned}
& \frac{d^{2} \Phi}{d g^{2}}=\frac{S-\Lambda \eta^{-1}(h S)^{1-\eta} \Phi}{(1-2 \eta) \Lambda \eta^{-1}(h S)^{1-\eta} g}<0, \text { if } \frac{1}{2}<\eta<1 \\
& \frac{d^{2} \Phi}{d g^{2}}=\frac{S-\Lambda \eta^{-1}(h S)^{1-\eta} \Phi}{(1-2 \eta) \Lambda \eta^{-1}(h S)^{1-\eta} g}>0 \text {, if } 0<\eta<\frac{1}{2} .
\end{aligned}
$$

Hence in case of $1 / 2<\eta<1$, the curve $S S$ is strictly concave and depicted by Figure 1-(a). However, if $1 / 0<\eta<1 / 2$, the curve $S S$ is strictly convex and depicted by Figure 1-(b). 
Figure 1. (a) The steady-state equilibrium (The case of $\theta=1,1 / 2<\eta<1$ ), (b) The steadystate equilibrium (The case of $\theta=1,0<\eta<1 / 2$ )

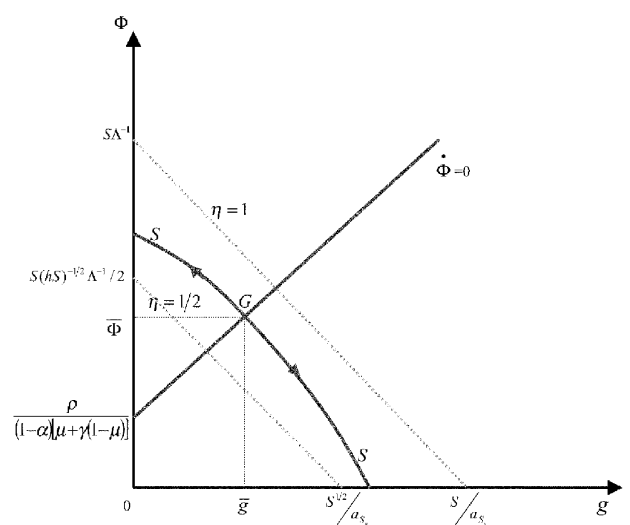

(a)

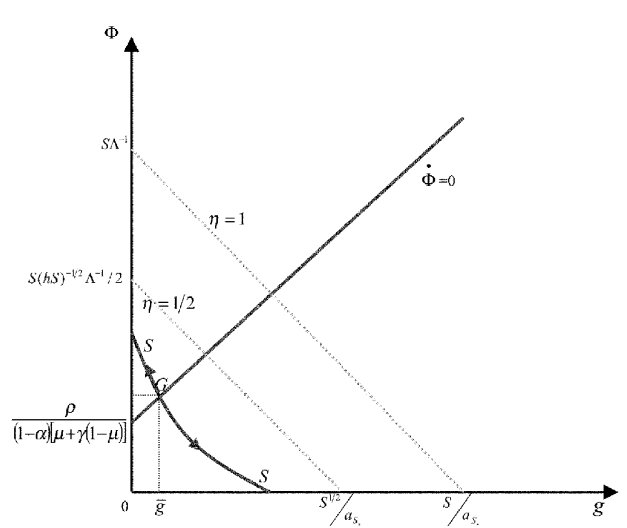

(b)

must be lower. Lower output implies higher prices, higher skilled wages, a greater value of the innovating firm, and thus a smaller inverse value of the stock market. The $\dot{\Phi}=0$ locus shows combinations of $\Phi$ and $g$ along which $\dot{\Phi}=0$. In other words, a rate of decline in the share price of the representative innovating firm that exactly matches the rate of new knowledge-based service development. From the no-arbitrage condition (A10) we see that a smaller aggregate value of the stock market (or larger inverse value) is associated with a faster rate of decline in firm value. Everywhere above the $\dot{\Phi}=0$ locus, the number of varieties grows less rapidly than the value of the typical firm falls (so $\Phi$ rises), while below the line the opposite is true. The arrows show the implied directions of movement along SS.

In the case where $S \eta(h S)^{\eta-1} / \Lambda>\rho /(1-\alpha)[\mu+\gamma(1-\mu)]$, the two curves must intersect in the positive orthant, as shown in the diagram. The intersection point is one which the dynamic forces in the economy effect no change in $\Phi$ and $g$ over time. That is, if the economy were to reach this point, then innovation would continue at a fixed rate, the division of resources among $R \& D$, knowledge-based service, manufacturing good and service remain constant through time, and the aggregate value of the stock market would not change. However, this expectations can be fulfilled only if the economy jumps immediately to this steady state. In the steady-state equilibrium represented by point $G$, knowledge-based service development continues indefinitely, always at a constant rate. Using the equations. $\dot{\Phi}=0$ and (22), we can calculate the steady-state rate of innovation implicitly,

$$
(1-\alpha)[\mu+\gamma(1-\mu)] a_{S_{n}}^{\frac{1}{\eta}} g^{\frac{1}{\eta}}+\Lambda \eta^{-1}(h S)^{1-\eta} g
$$




$$
=(1-\alpha)[\mu+\gamma(1-\mu)] S+\Lambda \eta^{-1}(h S)^{1-\eta} \rho
$$

With the steady-state rate of innovation, we have the following proposition.

Proposition 2 The steady-state rate of innovation g depends positively on the exponential parameter $\eta$ as well as the total skilled labor force (S), constant expenditure share of service $X(\mu)$, constant expenditure share of intermediate service $Z$ in the production of manufacturing $Y(\gamma)$, and negatively on the constant subjective discount rate $(\rho)$ and the inverse productivity parameter of $R \& D\left(a_{S_{n}}\right)$.

In this feature, the growth effect of scale can be eliminated by allowing for concavity in skilled labor devoted to R\&D in knowledge production function. However, lowered and sustained innovation is possible because the cost of knowledge-based service development falls with the accumulation of knowledge capital, even as the return to the marginal innovation declines. The nonappropriable benefits from R\&D keep the state of knowledge moving forward, and so the private incentives for further research are still maintained.

\section{Trade in Knowledge-based Services and Flows of Knowledge}

In this section we conduct a series of thought experiments. Despite the argument that the flows of knowledge can not easily be separated from trade in intermediate inputs, the growth effects of knowledge spillover and those of trade in intermediate inputs are conceptually distinct. ${ }^{7}$ Thus our specification permits a sharp distinction between trade in knowledge-based services and flows of knowledge. We start with two identical, completely isolated economies of the kind as specified above. To make the point most strongly, assume that the economies have identical tastes, technologies and factor endowments. We first allow for trade in knowledge-based services through digital markets as well as final outputs, but continue to restrict the flow of knowledge. In this specification, there is no intersectoral trade by assuming the same technologies and factor endowments. However, the only trades that take place are exchanges of knowledge-based services as intermediate inputs. To emphasize the distinction between knowledgebased service and knowledge, we assume that trade in knowledge-based services does not induce any transmission of knowledge. Under these assumptions we show that trade in knowledge-based services has no effect on the long-run rate of growth. The second examination is the effects of opening communications

\footnotetext{
${ }^{7}$ Feenstra (1996) argues that the diffusion of knowledge may be facilitated by trade in intermediate inputs.
} 
networks and permitting flows of knowledge in the absence of trade in knowledge-based services. There would be no incentive for researchers in different economies to specialize in different blueprints either before or after flows of knowledge are permitted. In this case, permitting the international transmission of knowledge would speed up worldwide growth rates to some extent. With free communication each researcher would be working with a larger stock of knowledge than would otherwise have been the case. In the third experiment, we consider the effect of permitting flows of knowledge in the presence of trade in knowledge-based services. In this integration, we show that allowing flows of knowledge results in a permanently higher growth rate.

\section{A. Trade in Knowledge-based Services without Flows of Knowledge}

This first example shows that the form of trade between the two economies is very simple. Because two economies are identical, all trades that take place are exchanges of knowledge-based services via digital marketplaces. Hence it is straight forward to show that opening trade has no permanent effect on the rate of growth.

In balanced endogenous growth without scale effects, the rate of growth of output is proportional to the rate of growth of $n, \dot{n} / n=S_{n}^{\eta} / a_{S_{n}}$, which is determined by the allocation of skilled labor $S=S_{y}+S_{z}+S_{n}$, among the manufacturing sector, the knowledge-based service sector, and the research sector.

Before trade is opened, the number of different types of intermediate services that are used in both the manufactures and the services must equal the number that has been designed and produced domestically. Along the new balanced growth path after trade is opened, the number of types of intermediate services used in each economy approaches twice the number that has been produced and designed domestically. In their pursuit of monopoly rents, researchers in the two economies will specialize in the production of different types of blueprints and avoid overlapping. Then the worldwide stock of designs will eventually be twice as large as the stock that has been produced in either economy.

With trade in the specialized knowledge-based services through digital markets, domestic manufacturings and services can take advantage of foreign varieties and vice versa. However, the level $z$ at which each knowledge-based service is used in each economy will return to the level that obtained under isolation. For the research sector, opening of trade implies that the market for any knowledge-based services is twice as large as it was in the absence of trade. However, the knowledge 
represented by $n^{*}$ is not available for use in research because flows of knowledge are not permitted. In this model free trade in knowledge-based services can affect the level of output and can thus affect welfare, but it does not affect long-run growth rates.

Consider the case in which two isolated economies start from completely nonintersecting sets of blueprints $n$ and $n^{*}$ that have the same measure. Before trade in knowledge-based services, the domestic economy will use intermediate inputs at the level $z$ for $n$ types of knowledge-based services and foreign economy will use intermediate inputs at the same level $z$ for $n^{*}$ different types of knowledgebased services. If existing intermediate service is freely mobile, each economy will immediately exchange half of its intermediate service stock for half of the intermediate service stock of the other economy when trade in knowledge-based service is allowed.

As in Figure 2, each will be using intermediate service at the level 1/2 on a set of intermediate services of measure $z+z^{*}$. From the functional form of production in manufacturings and services given in Equations (4) and (6), it follows that immediately after trade is opened. Output of manufacturings in each economy jumps by a factor of $2^{\gamma(1-\alpha) / \alpha}$, and output of services in each economy jumps by a factor $2^{(1-\alpha) / \alpha}$. This is analogous to the kind of level effect one encounters in the neoclassical model and in static models of trade with differentiated inputs in production. Hence we have the following proposition.

Proposition 3 The opening of world trade in knowledge-based services in the

Figure 2. Production and trade in knowledge-based services without flows of knowledge

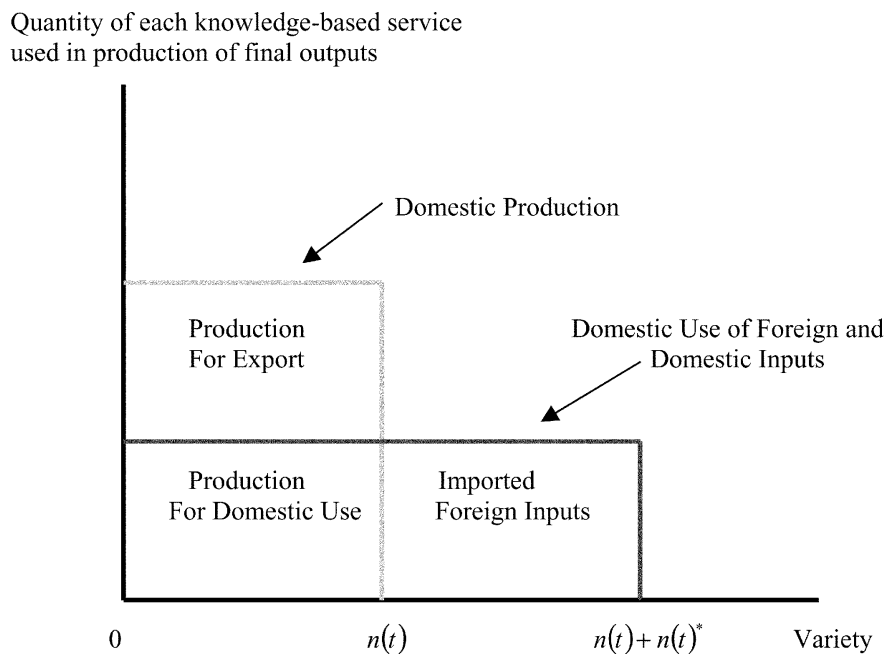


absence of flows of knowledge can affect the level of output, and can thus affect welfare. In each economy, output of services expands intensively by a factor $2^{(1-\alpha) / \alpha}$, output of manufacturing expands partially by a factor of $2^{\gamma(1-\alpha) / \alpha}$ which is depending on $\gamma$. However, this welfare gains has no permanent effect on the rate of growth.

If the two different economies start from a position with exactly overlapping sets of blueprints prior to the opening of trade, the timing of the effect on output is different, but the eventual effect is the same. The level of output at future times will generally be higher from what it would have been without trade in knowledgebased services. However, when the transitory effects have disappeared, the underlying growth rate will be the same as it was prior to the opening of trade in knowledge-based services.

\section{B. Flows of Knowledge without Trade in Knowledge-based Services}

Now we consider the alternative case in which flows of knowledge through digital markets are permitted but flows of final outputs and intermediate inputs are not. Let us assume for simplicity that the international exchange of knowledge takes place instantaneously and costlessly. Researchers in each economy learn from the experiments that are carried out abroad as well as from the R\&D projects undertaken locally. However, research endeavors that are common to the two economies contribute only once to the world stock of knowledge. In this case the results hinge on the degree of overlap between the set of ideas that are produced in each economy. In the absence of trade in knowledge-based services as well as final outputs, there would be no incentive for researchers in different economies to specialize in different designs either before or after flows of knowledge are permitted. With international diffusion of technical information, the knowledge stock in domestic is given by $n+\kappa n^{*}$, where $\kappa$ is the fraction of knowledge available in foreign that are not available in domestic. Notice that $n+\kappa n^{*}=n^{*}+\kappa n$, which represents the number of distinct research projects that have been conducted somewhere in the world.

The innovating firms introduce new varieties at a cost of $a_{S_{n}} v / \eta n S_{n}^{\eta-1}$ per blueprint, and so free entry implies that

$$
\phi_{n}=\frac{a_{S_{n}} v}{\eta\left(n+\kappa n^{*}\right) S_{n}^{\eta-1}}
$$

The international diffusion of knowledge through digital markets reduces the input requirement for $R \& D$. Then the skilled labor market clearing condition is 


$$
\left(a_{S_{n}} \frac{\dot{n}}{n+\kappa n^{*}}\right)^{\frac{1}{\eta}}+b_{S_{x}} \frac{\mu}{P_{x}}+b_{S_{y}} \frac{1-\mu}{P_{y}}=S
$$

Two further conditions characterize a steady state of the world economy with international knowledge diffusion. First, the value of the stock market in each economy remain constant. Second, the ratio of the numbers of blueprints available in each economy $n / n^{*}$ is constant. A constant ratio of blueprints ensures a constant allocation of resources to each economy's research sector when each has a constant rate of innovation. It requires convergence of the long-run innovation rates in the two economies; that is $g=g^{*}$. Then the steady-state no-arbitrage condition (23) implies that

$$
(1-\alpha)[\mu+\gamma(1-\mu)] \Phi=g+\rho
$$

where $\Phi$ denotes the inverse of the aggregate stock market value in each economy $\left(\Phi=\Phi^{*}\right)$.

We can use (5), (7), (25) and the definition of $\Phi$ to calculate the steady-state levels of employment in the final output sectors of each economy. They are

$$
\begin{aligned}
& \frac{1}{P_{x}}=\left(\frac{a_{S_{n}}}{\delta b_{L_{x}}+b_{S_{x}}}\right) \eta^{-1}(h S)^{1-\eta} \Phi\left(\frac{\dot{n}}{n+\kappa n^{*}}\right) \\
& \frac{1}{P_{y}}=\left(\frac{a_{S_{n}}}{\delta b_{L_{y}}+b_{S_{y}}}\right) \eta^{-1}(h S)^{1-\eta} \Phi\left(\frac{\dot{n}}{n+\kappa n^{*}}\right)
\end{aligned}
$$

We substitute (28) and (29) into (26) and use $g=g^{*}$ to derive

$$
\left(\frac{n}{n+\kappa n^{*}}\right)\left[a_{S_{n}}^{\frac{1}{\eta}} g^{\frac{1}{\eta}}\left(\frac{n}{n+\kappa n^{*}}\right)^{\frac{1-\eta}{\eta}}+\Lambda \eta^{-1}(h S)^{1-\eta} \Phi\right]=S
$$

Dividing this equation, as it applies to domestic economy, by the same equation applied to foreign economy, we find that the ratio of the numbers of blueprints available in each economy is equal in the long-run to the ratio of the sizes of the two skilled labor forces. Finally, we are ready to derive the long-run rate of innovation in the world economy with international knowledge spillovers but no knowledge-based service trade nor final outputs trade.

Using (30), we obtain

$$
a_{S_{n}}^{\frac{1}{\eta}} g^{\frac{1}{\eta}}\left(\frac{n}{n+\kappa n^{*}}\right)^{\frac{1-\eta}{\eta}}+\Lambda \eta^{-1}(h S)^{1-\eta} \Phi=S+\kappa S^{*}
$$


Equations (27) and (31) can be solved for $g$ and $\Phi$, which gives

$$
\begin{gathered}
(1-\alpha)[\mu+\gamma(1-\mu)] a_{S_{n}}^{\frac{1}{\eta}} g^{\frac{1}{\eta}}\left(\frac{n}{n+\kappa n^{*}}\right)^{\frac{1-\eta}{\eta}}+\Lambda \eta^{-1}(h S)^{1-\eta} g \\
=(1-\alpha)[\mu+\gamma(1-\mu)]\left(S+\kappa S^{*}\right)-\Lambda \eta^{-1}(h S)^{1-\eta} \rho
\end{gathered}
$$

When we compare (32) with (24), we see that the opening of flows of knowledge through digital markets accelerates innovation and growth in both economies. Research in any location contributes to a global stock of knowledge, which accumulates more rapidly than would the local stocks of knowledge in isolated research communities. The more rapid accumulation of knowledge implies a more rapid reduction in the cost of knowledge-based service development in each economy, and so the innovating firms introduce new varieties at a faster pace. At the same time it is clear from (32) that the benefits from flows of knowledge are attenuated by any duplication of research effort. Hence we have the following proposition.

Proposition 4 The fluid flow of knowledge in the absence of trade in knowledgebased services would speed up worldwide growth rates to some extent. However, the greater the extent of overlap in the research projects of the two economies (i.e., the smaller the $\kappa)$, the lower are the common long-run rates of innovation and growth.

\section{Flows of Knowledge with Trade in Knowledge-based Services}

Next we consider the integration of world product markets through digital marketplaces. In this experiments the integration of world final outputs and intermediate inputs markets with flows of knowledge suffices to eliminate research redundancy. Each new knowledge-based service invented in either economy in an equilibrium with world trade is a novel one. Thus each R\&D project contributes fully to the global stock of knowledge capital. Economic integration has two further effects. The opportunity to sell abroad enlarges the size of the market available to any producer. By itself this effect of trade tends to increase the profit opportunity available to any innovating firm. However, each innovator must compete for sales with a wider range of varieties. Such competition tends to reduce the profits available to any innovating firm. We must modify source of the equation from the above subsection in order to reflect the integration of world markets. Aggregate spending in the world economy equals $E+E^{*}$. This we normalize to be equal to one at every moment in time. The 
normalization guarantees a nominal interest rate of $\rho$ in each economy in a steady state with constant spending shares. Demand for the final outputs, manufacturings and services, in domestic economy varies now with the terms of trade. The advent of trade in knowledge-based services also alters the stock market value of profitmaking firms. Each innovating firm continues to set its price at a constant markup over marginal cost.

With international spillovers of knowledge capital and no overlap in research effort, the innovating firms have access to the global knowledge stock $n+n^{*}$. Therefore, the cost of developing a new knowledge-based service in domestic equals $a_{S_{n}} v / \eta\left(n+n^{*}\right) S_{n}^{\eta-1}$, and free entry ensures that

$$
\phi_{n}=\frac{a_{S_{n}} v}{\eta\left(n+n^{*}\right) S_{n}^{\eta-1}}
$$

Next, the expansion in knowledge capital reduces the skilled labor requirements for achieving a given rate of innovation. Final output producers also demand less skilled labor (at a given level of aggregate spending) due to the increased extent of market competition. The skilled labor market clearing conditions becomes

$$
\left(a_{S_{n}} \frac{\dot{n}}{n+n^{*}}\right)^{\frac{1}{\eta}}+b_{S_{x}} \frac{\mu}{P_{x}}+b_{S_{y}} \frac{1-\mu}{P_{y}}=S
$$

We concentrate on the steady state, which is characterized by constant aggregate values of each economy's stock market and by constant shares of each economy in the total number of differentiated varieties. For the value of each stock market to be constant, the share price of a typical firm must fall at the local rate of innovation. Constancy of the relative numbers of knowledge-based services requires convergence in long-run innovation rates. Thus the steady state noarbitrage condition (23) becomes

$$
(1-\alpha)[\mu+\gamma(1-\mu)] \Phi=g+\rho
$$

Equation (35) implies that in the long-run $\Phi$ is the same for both economies; in other words, the value of each economy's stock market is proportional to the share of world spending devoted to its final outputs. To solve for $g$, we need a second equation relating the rate of innovation to $\Phi$. This equation can be derived as follows:

$$
\left(\frac{n}{n+n^{*}}\right)\left[a_{S_{n}}^{\frac{1}{\eta}} g^{\frac{1}{\eta}}\left(\frac{n}{n+n^{*}}\right)^{\frac{1-\eta}{\eta}}+\Lambda \eta^{-1}(h S)^{1-\eta} \Phi\right]=S
$$


Since $\Phi$ is the same for both economies, (36) implies that the ratio of the number of varieties produced in each economy again reflects the relative sizes of the two skilled labor forces; that is, $n / n^{*}=S / S^{*}$.

We can apply (36) for domestic and foreign economy, and sum the results to obtain

$$
a_{S_{n}}^{\frac{1}{\eta}} g^{\frac{1}{\eta}}\left(\frac{n}{n+n^{*}}\right)^{\frac{1-\eta}{\eta}}+\Lambda \eta^{-1}(h S)^{1-\eta} \Phi=S+S^{*}
$$

The steady-state rate of innovation in the world economy with both knowledge diffusion and international trade can be obtained from (35) and (37) as follows

$$
\begin{gathered}
(1-\alpha)[\mu+\gamma(1-\mu)] a_{S_{n}}^{\frac{1}{\eta}} g^{\frac{1}{\eta}}\left(\frac{n}{n+\kappa n^{*}}\right)^{\frac{1-\eta}{\eta}}+\Lambda \eta^{-1}(h S)^{1-\eta} g \\
=(1-\alpha)[\mu+\gamma(1-\mu)]\left(S+S^{*}\right)-\Lambda \eta^{-1}(h S)^{1-\eta} \rho
\end{gathered}
$$

When we compare this rate to the steady state growth for an economy that does not engage in trade in knowledge-based services, but nonetheless captures all available international knowledge spillovers, we find that the marginal contribution of trade to long-run growth stems entirely from the elimination of duplicative research. Hence we have the following proposition.

Proposition 5 In the presence of trade in knowledge-based services, the fluid flow of knowledge would facilitate the long-run rate of innovation and growth worldwidely. In each economy, the more the knowledge stock is enhanced, the more productivity-enhancing new knowledge-based services is developed, and the more rapid innovation and growth can be done without any redundancy of research efforts.

If there happens to be no research overlapping in the equilibrium without trade $\kappa=1$, the integration of world markets will have no effect on the long-run growth rate in either economy.

\section{Conclusion}

The paper introduces a more sophisticated economic integration model of endogenous long-run growth without scale effects to provide in further detail the nexus of increased productivity in the advanced economies through economic integration via the digital marketplaces in the 1990s. In particular, we examine a general functional relationship of the knowledge production function and confirm that the process of endogenous innovation can only be self-sustaining with linear 
accumulation of knowledge capital. In a world in which economies are identical in terms of endowments and technologies, this analysis has addressed the relationship between economic integration and long-run growth performance. Depending on the form of a two-sector model of endogenous technological change in knowledge-based services, the integration could take the form of trade in knowledge-based services, flows of knowledge, or both.

The opening of world trade in the absence of flows of knowledge has no effect on long-run growth. However, there will be gains from intra-industry trades in knowledge-based services. This welfare gains come as 'level effects' rather than as 'growth effects'. The more flows of knowledge within and across national borders via Internet, the more knowledge stock is enhanced and the more productivity-enhancing new knowledge-based services it can develop, permitting sustained endogenous economic growth in a virtuous spiral. However, the benefits from flows of knowledge are attenuated by any overlapping of research effort in the absence of trade in knowledge-based services. Thus the greater the extent of overlapping in the research projects of the two economies, the lower are the common long-run rate of innovation and growth.

This result sheds light on the importance of free trade in knowledge-based services as well as the fluid flows of knowledge. That is, both knowledge diffusion and intra-industry trade in knowledge-based services can lead to the highest steady-state rate of innovation in the world economy. In this two-sector model, the intermediate services are used not only most intensively in the service sector, but in the 'service' function of manufacturing sector. Hence the more knowledgebased services are intensively used in the service sector and the more knowledgebased services are embedded in manufacturing sector, the more long-run endogenous growth each economy can achieve.

This analysis explains that the trade of knowledge-based services as well as the flow of knowledge through the digital marketplaces have played an important role in facilitating further growth in both service and manufacturing sectors in the 1990s. These findings suggest that removing restrictions on trade in knowledgebased services and fluid flows of knowledge should be a high priority issue in the current trade policy discussions.

Received 25 November 2001, Accepted 23 December 2002 
Economic Integration with Endogenous Technological Change in Knowledge-based Services 73

\section{References}

Aghion, P. and P. Howitt (1992), "A Model of Growth through Creative Destruction," Econometrica 60, 323-351.

Ark, B. van, E. Monnikhof, and N. Mulder (1999), "Productivity in Services: An International Comparative Perspective," Canadian Journal of Economics 32, 471-499.

Chang, W.W. (1973), "Intermediate Products, Aggregation, and Economic Growth," The Southern Economic Journal 40(1), 56-65.

Choi, S.-Y. and A.B. Whinston (2000), The Internet Economy: Technology and Practice, SmatEcon Publishing, Austin, Taxas.

Feenstra, R.C. (1996), "Trade and Uneven Growth," Journal of Development Economics 49, 229-256.

Grossman, G.M. and E. Helpman (1991), Innovation and Growth in the Global Economy, Cambridge, Mass.: MIT Press.

Howitt, P. (1999), "Steady Endogenous Growth with Population and R\&D Inputs Growing," Journal of Political Economy 107, 715-730.

Jones, C.I. (1995), "R\&D-based Models of Economic Growth," Journal of Political Economy 103, 759-84.

Jones, C.I. (1999), "Growth: with or without Scale Effects?," American Economic Review 89, 139-144.

Lehr, F. and F. Lichtenberg (1999), "Information Technology and its Impact on Productivity: Firm-Level Evidence from Government and Private Data Sources, 1977-1993," Canadian Journal of Economics 32, 335-362.

Licht, G. and D. Moch (1999), "Innovation and Information Technology in Services," Canadian Journal of Economics 32, 363-383.

OECD (1999), Managing National Innovation Systems, OECD, Paris.

OECD (2000), A New Economy?: The Changing Role of Innovation and Information Technology in Growth, OECD, Paris.

Rivera-Batiz, L.A. and P.M. Romer (1991), "Economic Integration and Endogenous Growth," Quarterly Journal of Economics 106, 531-555.

Romer, Paul, M. (1990), "Endogenous Technological Change," Journal of Political Economy 98, S71-S102.

Segerstrom, P.S. (1998), "Endogenous Growth without Scale Effects," American Economic Review 88, 1290-1310.

Wolfson, M.C. (1999), "New Goods and the Measurement of Real Economic Growth," Canadian Journal of Economics 32, 447-470.

Yoon, S.-C. (2001), Essays on International Trade in Knowledge-based Services, Ph.D. dissertation (State University of New York at Buffalo, NY).

Young, A. (1998), "Growth without Scale Effects," Journal of Political Economy 106, 41-63. 


\section{Appendix}

In this Appendix we provide a reduced-form system that describes the equilibrium dynamics under a general knowledge production function by considering market clearing conditions in both factor and final output markets.

The unskilled labor market clearing condition is

$$
b_{L_{x}} \bar{X}+b_{L_{y}} \bar{Y}=L
$$

and the skilled labor market clearing condition is

$$
\left[\frac{a_{S_{n}}}{n^{\theta-1}} \frac{\dot{n}}{n}\right]^{\frac{1}{n}}+b_{S_{x}} \bar{X}+b_{S_{y}} \bar{Y}=S
$$

where $\bar{X} \equiv n^{-\sigma / \gamma} X, \bar{Y}=n^{-\sigma} Y$.

Using the output market clearing conditions, we can get

$$
\mu \frac{E}{P_{x}}=\bar{X}
$$

and

$$
(1-\mu) \frac{E}{P_{y}}=\bar{Y}
$$

and from Equations (4), (6), (A3), and (A4) with the Cobb-Douglas system, we can also obtain

$$
\frac{[\mu+\gamma(1-\mu)] E}{q}=Z
$$

In this analysis, it proves convenient to define $w(t)=\delta(t) v(t)$ with $0<\delta(t)<1$ at time $t$. In equilibrium $\delta$ is constant. We assume that a fraction $h(t)$ of the total skilled labor force $S$ works in R\&D so that $S_{n}=h(t) S$, with $0<h(t)<1$ at time $t$. In equilibrium, $h$ is constant. We can also simplify two new variables by using $\Phi \equiv 1 / n \phi_{n}$ to represent the inverse of aggregate equity value of the R\&D sector and $\dot{g} \equiv \dot{n} / n$ to denote the instantaneous rate of 'innovation' in the economy (that is, the rate at which new varieties are being introduced). Hence, we can solve for equilibrium output and factor prices and sectoral output levels all as a function of $\Phi$. We denote these functional relationships by $v(\Phi), P_{x}(\Phi), P_{y}(\Phi), p_{z}(\Phi), \bar{X}(\Phi), \bar{Y}(\Phi)$.

Since the cost of a blueprint is $a_{S_{n}} v / \eta n^{\theta} S_{n}^{\eta-1}$, the free entry condition implies

$$
a_{S_{n}} \nu / \eta n^{\theta} S_{n}^{\eta-1} \geq \phi_{n}
$$


with equality whenever $\dot{n}>0$. We impose a normalization of prices that makes nominal spending constant through time. With

$$
E(t)=1 \text { for all } t
$$

equation (3) implies that

$$
r(t)=\rho \text { for all } t
$$

Using the pricing equations (5), (7), the factor market clearing condition (A2) with the output market clearing conditions (A3) and (A4), and the free entry condition (A6), we can prescribe the evaluation of the number of varieties as follows

$$
\frac{\dot{n}}{n}=\left[S-\Lambda \eta^{-1}(h S)^{1-\eta} n^{\theta-1} \Phi\right]^{\eta} \frac{n^{\theta-1}}{a_{S_{n}}} \equiv e^{n}(n, \Phi)>0 \text { if } \Phi<\left(\frac{1}{\Lambda}\right) \eta h^{\eta-1} S^{\eta} n^{\theta-1}
$$

where $\Lambda=\frac{\mu a_{S_{n}} b_{S_{x}}\left(\delta b_{L_{y}}+b_{S_{y}}\right)+(1-\mu) a_{S_{n}} b_{S_{y}}\left(\delta b_{L_{x}}+b_{S_{x}}\right)}{\left(\delta b_{L_{y}}+b_{S_{y}}\right)\left(\delta b_{L_{x}}+b_{S_{x}}\right)}$.

Then we substitute the profit rate (12) with (A5) and equation (A8) into the noarbitrage condition (14) to derive an equation for the change in firm value as a function of the current value of a blueprint and the number of available blueprints. With equation (A7), the result is

$$
\frac{\dot{\phi}_{n}}{\phi_{n}}=\rho-\frac{(1-\alpha)[\mu+\gamma(1-\mu)]}{n \phi_{n}}
$$

and the definitions of $\Phi$ and $g$ imply that

$$
\frac{\dot{\Phi}}{\Phi}=-g-\frac{\dot{\phi}_{n}}{\phi_{n}}
$$

Thus, the value of the typical blueprint evolves according to (A10), which, with (A9) and (A11), implies that

$$
\frac{\dot{\Phi}}{\Phi}=(1-\alpha)[\mu+\gamma(1-\mu)] \Phi-\rho-\left[S-\Lambda \eta^{-1}(h S)^{1-\eta} n^{\theta-1} \Phi\right]^{\eta} \frac{n^{\theta-1}}{a_{S_{n}}} \equiv e^{\Phi}(n, \Phi)
$$

Equations (A9) and (A12) constitute an autonomous system of differential equations. They apply whenever the implied rate of knowledge-based service development is nonnegative. 
Figure A1. The stationary equilibrium (The case where $\theta<1$ )

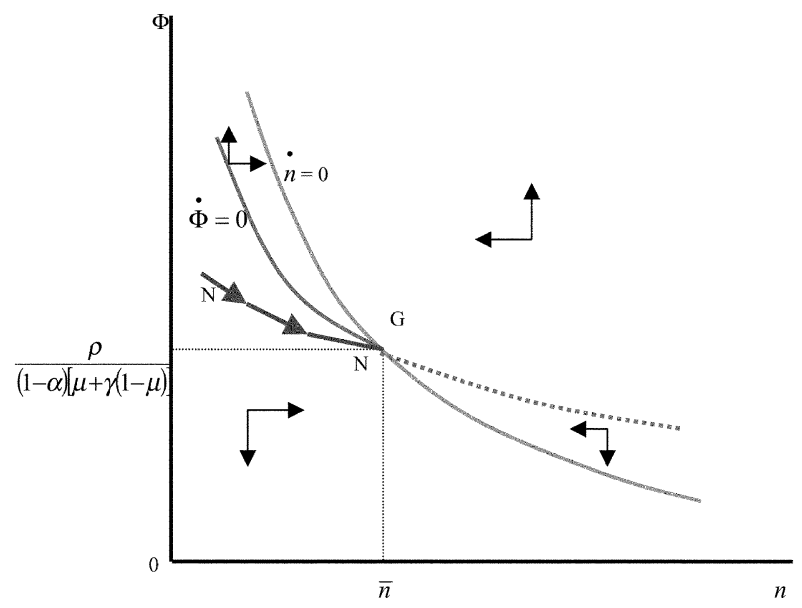

First, we investigate the case where $\theta<1$. This is the case of decreasing returns in the accumulation of knowledge. Figure A1 depicts the phase diagram for the system in this case. In Figure A1, the region above the $\dot{n}=0$ locus is infeasible since $\dot{n}$ is not allowed to be negative in view of (10). The $\dot{\Phi}=0$ locus shows the combinations of these variables along which the aggregate value of the stock market does not change. This locus slopes downward. Two curves intersect in the positive orthant. ${ }^{8}$ The stationary equilibrium for this economy is depicted by point $G$. If the initial number of brands exceeds $\bar{n}$, then the economy enters a stationary state immediately. If the economy inherits less than $\bar{n}$ brands, then point $G$ is approached along the (saddle path) trajectory labeled $N N$. In either event no

${ }^{8}$ With $\theta<1$, we have

$$
\left.\frac{d \Phi}{d n}\right|_{n=0}=-\frac{\Theta_{n}}{\Theta_{\Phi}}=\frac{(\theta-1) \Phi}{n}<0
$$

and

where

$$
\left.\frac{d \Phi}{d n}\right|_{\Phi=0}=-\frac{\Omega_{n}}{\Omega_{\Phi}}=\frac{(\theta-1) \Phi}{n}\left(\frac{\Phi \Xi}{\Phi \Xi+\rho}\right)<0
$$

$$
\Xi=\frac{1}{a_{S_{n}}}\left[S-\Lambda \eta^{-1}(h S)^{1-\eta} n^{\theta-1} \Phi\right]^{\eta-1}\left[(\eta-1) \Lambda \eta^{-1}(h S)^{1-\eta}+\frac{S n^{\theta-1}}{\Phi}\right] .
$$

The $\dot{n}=0$ and $\dot{\Phi}=0$ curves are both negatively sloped. Since $\rho>0$, we obtain

$$
-\frac{\Theta_{n}}{\Theta_{\Phi}}>-\frac{\Omega_{n}}{\Omega_{\Phi}} .
$$

Thus the $\dot{n}=0$ locus is steeper than the $\dot{\Phi}=0$ locus, as depicted in Figure A1. 
Figure A2. The stationary equilibrium (The case where $\theta>1$ )

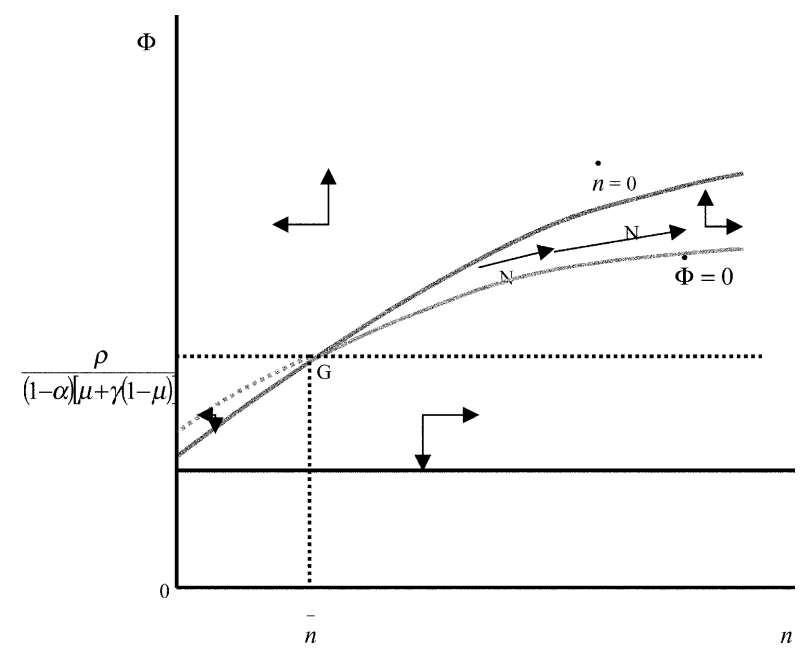

innovation takes place in the long run. We see that if returns to R\&D in the production of knowledge are decreasing and the contribution of new R\&D to the stock of knowledge becomes sufficiently small, then the long-run stagnation is inevitable, just as it is in the absence of knowledge spillovers. The arrows of motion suggest that the steady-state equilibrium point is a saddle point (see the proof). $|J|$ is negative since $\theta<1$. Thus the roots are of opposite signs, and the steady-steady point $(\bar{n}, \bar{\Phi})$ is a saddle point equilibrium.

Second, we consider the possibility that $\theta>1$. That is the case of an increasing function of $n$. Increasing returns in the production of knowledge is necessary but not sufficient for this to be so. We suppose also that 1) $(1 / \Lambda) \eta h^{\eta-1} S^{\eta} n^{\theta-1}<\rho /(1-$ $\alpha)[\mu+\gamma(1-\mu)], 2)(1 / \Lambda) \eta h^{\eta-1} S^{\eta} n^{\theta-1}>\rho /(1-\alpha)[\mu+\gamma(1-\mu)]$ for $n$ sufficiently large, and 3) $n^{\theta-1}$ is bounded from above.

Figure A2 depicts this case. ${ }^{9}$ Conditions 1) and 2) ensure the existence of an intersection of $\dot{n}=0$ locus and the $\dot{\Phi}=0$ locus at $\Phi=\rho /(1-\alpha)[\mu+\gamma(1-\mu)]$ in the positive orthant. Let $\bar{n}$ be the number of varieties at equilibrium. The equilibrium point $(\bar{n}, \bar{\Phi})$ is unstable node, since $|J|>0$ and trace $J>0$ if $\theta>1$ (see the proof). If the initial $n>\bar{n}$, there are paths that are everincreasing in $n$ and

${ }^{9}$ With $\theta>1$, we have $\left.\frac{d \Phi}{d n}\right|_{n=0}>0$ and $\left.\frac{d \Phi}{d n}\right|_{\dot{\Phi}=0}>0$. Thus the $\dot{n}=0$ and $\dot{\Phi}=0$ curves are both positively sloped. Since $\rho>0$, we ahve

$$
-\frac{\Theta_{n}}{\Theta_{\Phi}}>-\frac{\Omega_{n}}{\Omega_{\Phi}} .
$$


$\Phi$ if they don't cross the $\dot{\Phi}=0$ locus. If the initial $n<\bar{n}$ and given that $\dot{n} \geq 0$, then $\Phi$ decreases and $n$ increases over time, eventually $\Phi \rightarrow 0$ as $t \rightarrow \infty$. All other feasible paths again will have $n \rightarrow \infty$ and $\Phi \rightarrow 0$ as $t \rightarrow \infty$. Thus unless the initial $n$ is equal to $\bar{n}$, no steady-state exists in the model. If $n=\bar{n}$, the steady-state $\Phi$ is equal to $\rho /(1-\alpha)[\mu+\gamma(1-\mu)]$.

Third, we check the case in which $\theta=1$ for all $n$. In this case, a steady-state equilibrium exists under some conditions and the growth rate depends on research intensity.

Proof. We provide a proof of the dynamic stability of equilibrium by examining the signs of the roots of the linear version of the coefficient matrix. The differential equation system in (A9) and (A12) is of the form

$$
\begin{aligned}
& \dot{n}=n e^{n}(n, \Phi)=\Theta(n, \Phi) \\
& \dot{\Phi}=\Phi e^{\Phi}(n, \Phi)=\Omega(n, \Phi)
\end{aligned}
$$

Let $J$ be the coefficient matrix of the linearized version of this system in the neighborhood of the steady-state equilibrium:

$$
J \equiv\left[\begin{array}{ll}
\partial \Theta / \partial n & \partial \Theta / \partial \Phi \\
\partial \Omega / \partial n & \partial \Omega / \partial \Phi
\end{array}\right]
$$

Using (A9) and (A12), we have

$$
\begin{aligned}
J & \equiv\left[\begin{array}{cr}
(\theta-1) \Lambda \eta^{-1}(h S)^{1-\eta} n^{-\theta} \Phi & -\Lambda \eta^{-1}(h S)^{1-\eta} n^{1-\theta} \\
0 & (1-\alpha)[\mu+\gamma(1-\mu)]
\end{array}\right] \\
& =(\theta-1)(1-\alpha)[\mu+\gamma(1-\mu)] \Lambda \eta^{-1}(h S)^{1-\eta} n^{-\theta} \Phi
\end{aligned}
$$

Thus we obtain the following relationship:

$$
|J|\left\{\begin{array}{l}
< \\
= \\
>
\end{array}\right\} \quad \text { if } \theta=\left\{\begin{array}{l}
< \\
= \\
>
\end{array}\right\} 1
$$

If $\theta<1$, then $|J|$ is negative. Thus the roots are of opposite signs, and the steady-steady point $(\bar{n}, \bar{\Phi})$ is a saddle point equilibrium. If $\theta>1$, then $|J|$ is positive and trace $J>0$. Thus the equilibrium point $(\bar{n}, \bar{\Phi})$ is unstable node. 